\title{
Initial Experience of Applying TWIST Dixon with Flexible View Sharing in Breast DCE-MRI
}

Yuan Le $\mathrm{PhD}^{1}$, Hal D. Kipfer $\mathrm{MD}^{1}$, Dominik M. Nickel $\mathrm{PhD}^{2}$, Randall Kroeker $\mathrm{PhD}^{2}$, Brian Dale $\mathrm{PhD}^{2}$, Stephanie P. Holz MD${ }^{1}$, Elisabeth Weiland $\mathrm{PhD}^{2}$, Chen Lin $\mathrm{PhD}^{1}$.

1. Department of Radiology and Imaging Science, Indiana University School of Medicine, Indianapolis, IN, United States

2. Siemens Healthcare, Erlangen, Germany

Running Head: Breast DCE-MRI with TWIST Dixon and flexible view sharing

Correspondence:

Chen Lin, PhD

Department of Radiology and Imaging Science

Indiana University School of Medicine

950 West Walnut St. R2 E124

Indianapolis, IN 46202

Email: clin1@iupui.edu

Phone: (317) 278-6572

This is the author's manuscript of the article published in final edited form as:

Le, Y., Kipfer, H. D., Nickel, D. M., Kroeker, R., Dale, B. M., Holz, S. P., ... Lin, C. (2016). Initial Experience of Applying TWIST-Dixon With Flexible View Sharing in Breast DCE-MRI. Clinical Breast Cancer, 16(3), 202-206.

http://doi.org/10.1016/j.clbc.2015.11.007 
Initial Experience of Applying TWIST Dixon with Flexible View Sharing in Breast DCE-MRI

Word count: 2578 


\begin{abstract}
:
Objective: To develop a new fast imaging technique with flexible TWIST view sharing to achieve variable temporal resolution and with flexible echo time Dixon to achieve robust fat suppression and to evaluate its application in breast DCE-MRI.
\end{abstract}

Materials and Methods: TWIST Dixon technique was improved with more flexible view sharing and echo times (TWIST Dixon Flex). In a dynamic series each measurement can be separately prescribed as "Full”, "Partial” or "Center-only”. The spatial and temporal resolution can then be adjusted throughout the measurements to match the dynamic characteristics of contrast enhancement at different phases. The potential advantages of TWIST Dixon Flex were evaluated with 18 cases of the clinical breast DCE MRI. The maximum contrast uptake slope was measured based on the measurements with high temporal resolution during in early arterial phase and the morphological features of contrast enhancement were captured near peak enhancement with high spatial resolution.

Results: Based on the initial results of five clinical cases, TWIST Dixon Flex technique is capable of detecting the difference in the rapid contrast uptake from different region of breast tumor which is not possible in conventional breast DCE-MRI. The quality of post-contrast TWIST Dixon Flex images is superior to the conventional fat suppressed images.

Conclusion: The new TWIST Dixon Flex technique provides potentially valuable information of the early tumor enhancement, while maintaining excellent image quality at peak and post-contrast enhancement. This technique could allow us to overcome the compromise on spatial over temporal resolution in clinical breast imaging.

Key Words: Breast Cancer, Dynamic Contrast Enhance MRI, TWIST, Dixon, View Sharing 


\section{Introduction}

Recently, Time-resolved Angiography with Stochastic Trajectories (TWIST), an acceleration technique based on view sharing, has been receiving increasing attention in breast DCE-MRI applications (1-5). When combined with the dual-echo Dixon fat and water separation technique, TWIST-Dixon provides superior fat suppression and higher SNR efficiency to allow better overall image quality for breast DCE-MRI applications (4).

Krishnan et al (6) demonstrated that $k$-space data acquisition can be optimized according to the spatial-temporal bandwidth-based analysis and, by prioritizing the data acquisition based on the spectral energy of corresponding $k$-space data, the fidelity of the images can be optimized. These results suggest that one can further improve dynamic acquisition by acquiring less peripheral $k$ space data during the period of rapid contrast wash-in to achieve higher temporal resolution, and by acquiring more peripheral $k$-space data during the delayed phase to obtain higher spatial resolution. The purpose of this work, therefore, is to improve the TWIST-Dixon technique by using a more flexible view sharing strategy (TWIST-Dixon-Flex), so that the peripheral $k$-space to be re-acquired in each measurement can be prescribed individually to allow high temporal resolution during the early contrast wash-in; and high spatial resolution at the peak enhancement. We have implemented and applied the improved TWIST-Dixon-Flex technique to breast DCEMRI of a small group of clinical patients with encouraging results.

\section{Methods}

With the standard TWIST view sharing technique (7), the $k$-space is divided into two parts: a central region (A) and a peripheral region (B). The peripheral $k$-space region is then further divided into several subsets $\left(B_{1} B_{2} \ldots B_{n}\right)$. In a dynamic acquisition with multiple measurements, the full $k$-space $\left(\mathrm{AB}_{1} \mathrm{~B}_{2} \ldots \mathrm{B}_{\mathrm{n}}\right)$ is sampled either at the beginning or the end of the whole series of 
measurements; for other measurements, the central $k$-space region and only one of the peripheral $k$-space subsets $\left(\mathrm{AB}_{\mathrm{i}}\right)$ are acquired. The images of each measurement are then reconstructed with missing $k$-space data 'shared' from the measurements in either forward or backward direction.

In the prototype TWIST-Dixon-Flex sequence, the sampling of $k$-space for each measurement can be prescribed individually as 'Full' acquisition with all $k$-space data acquired $\left(\mathrm{AB}_{1} \mathrm{~B}_{2} \ldots \mathrm{B}_{\mathrm{n}}\right)$; 'Partial' acquisition which is the same as standard TWIST acquisition ( $\left.\mathrm{AB}_{\mathrm{i}}\right)$; or 'Center-only' acquisition in which only the central $k$-space data are acquired (A). Missing $k$-space data will be shared from the nearest available measurements with backward sharing preferred. Such flexibility allows for variable temporal resolution during different stages of dynamic contrast enhancement. For example, for breast DCE-MRI performed in this study, one possible DCEMRI protocol would include one measurement with 'Full' acquisition before contrast injection, six measurements of 'Center-only' acquisition during the first 60 seconds of contrast wash-in and three additional measurements (two 'Partial' acquisitions followed with one 'Full' acquisition) starting from the estimated peak enhancement time point. Figure 1 shows the timeline of this mixed Full/Center-only/Partial/Full acquisition. Two gradient echoes were generated with bipolar readout gradients per TR to allow the separation of fat and water signals with flexible echo time Dixon.

To confirm the efficacy of TWIST Dixon with flexible view sharing for breast DCE-MRI, clinical evaluation was carried out with the approval of institutional review board. Eighteen patients (ages 35-62) scheduled for breast MRI exams were recruited and informed consent was obtained. Breast MRI exams were performed on a 1.5 T clinical MRI scanner (Aera, Siemens Medical Solution, Erlangen, Germany) using an eight channel Sentinelle breast coil (Hologic, Bedford, MA). The patients were scanned in head-first prone position. Imaging protocol 
included: (1) 3 plane localizer; (2) 3D T1 weighted prototype TWIST-Dixon-Flex sequence with a total of 10 measurements (1 pre-contrast, 6 between contrast injection and the estimated peak enhancement, and 3 post-contrast). A single dose of contrast agent $(0.1 \mathrm{mmol} / \mathrm{kg}$, ProHance, Bracco Diagnostics Inc, Princeton, NJ) was infused at $2 \mathrm{~mL} / \mathrm{s}$ and flushed with $20 \mathrm{~mL}$ saline immediately after the pre-contrast measurement. (3) 3D T1 weighted VIBE with SPAIR fat suppression, and (4) 3D T2 weighted data set with SPAIR fat suppression. The imaging parameters for TWIST-Dixon-Flex and VIBE-SPAIR are listed in Table 1. The in-phase images acquired with TWIST-Dixon-Flex before contrast injection also served as the non-fat-suppressed T1 weighted images typically included in our clinical breast MRI protocol. The maximum slope of initial contrast uptake was visualized and evaluated with the non-product prototype TWIST Breast Viewer software (Siemens Medical Solution, Erlangen, Germany).

The $1^{\text {st }}$ post-contrast set of TWIST-Dixon-Flex images (at peak enhancement) and the VIBESPAIR images acquired immediately after were reviewed by two radiologists specialized in breast imaging. The radiologists were blind of the diagnosis and clinical background when they performed the evaluation. Image quality criteria: Perceived SNR (P.SNR), Visualization of Anatomy, Fat Suppression Quality, Artifacts, and Overall Image Quality (IQ), were independently scored on a scale of $1-5$, with 1-poor, 2-below average, 3-average, 4-above average and 5-excellent.

To investigate the effect of the pulse sequence on image quality, ANOVA tests were performed. In each case the model tested the fixed effect of the pulse sequence, TWIST_DIXON vs. VIBE_SPAIR, plus the random effects of the reviewer and patients, on the specified image quality measure.

\section{Results}


Among the first five subjects enrolled in the study, one had DCIS, two had invasive ductal carcinoma and two had no detectable lesion.

Figure 2 shows the eight MIPs of measurement 3 - 10 in one of the patients with invasive ductal carcinoma (2a-2h). The enhancing tumor became visible starting from the $5^{\text {th }}$ measurement, which was acquired during the contrast wash-in. It also shows the overall enhancement start to decrease between the $9^{\text {th }}$ and $10^{\text {th }}$ measurement, signifying contrast wash-out. The maximum slopes of initial enhancement are similar for voxels 2 and 3 displayed as 'green' (less than 6\% enhancement per second) in the tumor. However, their enhancement curve types were opposite. Different regions in the tumor also reached the peak enhancement at different time points: voxel 1 (shown by arrow in $2 \mathrm{i}$ and curve in $2 \mathrm{j}$ ) reached the peak enhancement at the $7^{\text {th }}$ measurement; while voxel 2 peaked at the $9^{\text {th }}$ measurement (curve in $2 \mathrm{k}$ ), and voxel 3 was increasingly enhanced during the process (curve in 2l). With conventional imaging techniques, the peak enhancement of voxel 1 may have been missed.

Table 2 summarizes the image quality scores. Based on a paired T-test, TWIST-Dixon-Flex images have significantly higher scores than VIBE-SPAIR on all criteria. With higher SNR, better visualization of anatomy, superior fat suppression and less artifact, the images produced by TWIST-Dixon-Flex technique have higher overall image quality than those produced with the VIBE-SPAIR technique. These results are further demonstrated in Figure 3 with direct comparison of TWIST-Dixon-Flex and VIBE-SPAIR images from the same slice in one of the subjects.

\section{Discussions and Conclusions}

Our preliminary study showed that the TWIST-Dixon-Flex technique produces high spatial resolution images at the peak enhancement and afterward with image quality that is superior to 
the conventional VIBE-SPAIR technique. This is consistent with the results of a previous study comparing TWIST Dixon with VIBE-SPAIR at 3T (4). At the same time, TWIST-Dixon-Flex also provides rapid measurements during the period of contrast wash-in to evaluate the early arterial phase of contrast enhancement. With flexible view sharing, TWIST-Dixon-Flex is capable of measuring both the temporal characteristics and the morphological features of contrast enhancement of breast lesions, while providing accurate fat suppression and high SNR efficiency.

Fast acquisition with TWIST view sharing, but without Dixon fat suppression, was previously performed by Hermann et al (3): In comparison, the TWIST-Dixon-Flex imaging protocol in the current study has a larger FOV and higher spatial resolution. The resulting increased matrix size necessitates lower temporal resolution. The temporal resolution during the contrast wash-in, although lower than the previous study (3), appears to be adequate for measuring and differentiating maximum slope of initial contrast enhancement. One key difference is that TWIST-Dixon-Flex allows multiple measurements of different temporal resolution for the entire DCE-MRI through a single pulse sequence instead of a combination of several sequences.

A recent study by Mann et al (5) found that the detected maximum slope during the rapid contrast wash-in provides important information for lesion detection and classification. Therefore the fast Center-only measurements during that period of time can be very helpful. Possible errors in the TWIST-Dixon-Flex technique are temporal-spatial blurring and ringing artifacts as the results of view sharing. However, the TWIST-Dixon-Flex technique still provided images of higher overall quality than those acquired with conventional techniques. Our previous simulation work also showed that the error caused by such view sharing was comparable or less than that caused by the long acquisition time without view sharing (8). 
Another limitation is that the current prototype two-point Dixon fat and water separation algorithm fails occasionally, causing the fat and water signals in certain regions to be incorrectly assigned to fat-only and water-only images. The water signal of the pectoralis and the blood in aorta and ventricles were incorrectly assigned to the fat-only image in one or two measurements of some patients. However, this artifact has not been seen in the breasts.

Our initial experience is based on a small sample size and some of the analysis is qualitative. The clinical nature of the study also precludes more than one DCE-MRI image acquisition on the same patient. Therefore, the comparison we made is between the TWIST-Dixon-Flex images acquired at peak enhancement and the conventional VIBE-SPAIR images acquired immediately after the last TWIST-Dixon-Flex measurement. The delay may skew the results of the comparison. Our continuing study will include more clinical cases and different types of lesions so that the correlation between the early arterial enhancement characteristics and the type of lesion can be investigated and a more comprehensive image quality evaluation will be possible.

\section{Acknowledgements}


Table 1. Imaging Parameters of TWIST-Dixon-Flex and VIBE-SPAIR

\begin{tabular}{|c|c|c|}
\hline & TWIST-Dixon-Flex & VIBE-SPAIR \\
\hline Matrix & \multicolumn{2}{|c|}{$448 \times 358$} \\
\hline FOV (mm) & \multicolumn{2}{|c|}{$326-380$} \\
\hline Acceleration (GRAPPA) & \multicolumn{2}{|c|}{2} \\
\hline Number of Slices & \multicolumn{2}{|c|}{128} \\
\hline Slice Thickness (mm) & \multicolumn{2}{|c|}{1.5} \\
\hline Slice Resolution & \multicolumn{2}{|c|}{$79 \%$} \\
\hline Flip Angle (degree) & 15 & 15 \\
\hline Receiver BW (Hz/pix) & 400 & 400 \\
\hline TR / TE1 / TE2 (ms) & $6.6 / 2.23 / 4.02$ & $4.13 / 1.5$ \\
\hline Time per measurement (sec) & 8.9 / 62 / 116* & 66 \\
\hline Central k-space (A) size & $8 \%$ & N/A \\
\hline Peripheral k-space (B) sampling density & $50 \%$ & N/A \\
\hline
\end{tabular}

* For TWIST-Dixon-Flex, the time per measurement is for Center-Only / Partial / Full.

** SPAIR fat suppression module was applied once every 128 TRs. 
Table 2. Statistical Analysis of the Imaging Quality Scores

\begin{tabular}{llllll}
\hline & P. SNR & $\begin{array}{l}\text { Anatomy } \\
\text { Visualization }\end{array}$ & FS Quality & Artifact & Overall IQ \\
\hline TWIST-Dixon-Flex* & 3.9 & 4.06 & 4.47 & 4.04 & 4.23 \\
VIBE-SPAIR & 3.15 & 3.04 & 3.02 & 3.33 & 3.16 \\
Sequence p-val & $<0.0001^{* *}$ & $<0.0001^{* *}$ & $<0.0001^{* *}$ & $<0.0001^{* *}$ & $<0.0001^{* *}$ \\
\hline
\end{tabular}

* Mean Score

** TWIST-Dixon-Flex scores significantly higher than VIBE-SPAIR 


\section{References}

1. Janka R, Wenkel E, Geppert C, Kiefer B, Uder M. Breast MRI using TWIST: Doubling the spatial resolution "for free". International Society of Magnetic Resonance in Medicine scientific meeting. Stockholm, Sweden; 2010. p 4553.

2. Tudorica LA, Oh KY, Roy N, Kettler MD, Chen Y, Hemmingson SL, Afzal A, Grinstead JW, Laub G, Li X, Huang W. A feasible high spatiotemporal resolution breast DCE-MRI protocol for clinical settings. Magn Reson Imaging 2012;30(9):1257-1267.

3. Herrmann KH, Baltzer PA, Dietzel M, Krumbein I, Geppert C, Kaiser WA, Reichenbach JR. Resolving arterial phase and temporal enhancement characteristics in DCE MRM at high spatial resolution with TWIST acquisition. J Magn Reson Imaging 2011;34(4):973982.

4. Le Y, Kipfer H, Majidi S, Holz S, Dale B, Geppert C, Kroeker R, Lin C. Application of time-resolved angiography with stochastic trajectories (TWIST)-Dixon in dynamic contrast-enhanced (DCE) breast MRI. J Magn Reson Imaging 2013;38(5):1033-1042.

5. Mann RM, Mus RD, van Zelst J, Geppert C, Karssemeijer N, Platel B. A Novel Approach to Contrast-Enhanced Breast Magnetic Resonance Imaging for Screening: High-Resolution Ultrafast Dynamic Imaging. Invest Radiol 2014.

6. Krishnan S, Chenevert TL. Spatio-temporal bandwidth-based acquisition for dynamic contrast-enhanced magnetic resonance imaging. J Magn Reson Imaging 2004;20(1):129137.

7. Lim RP, Shapiro M, Wang EY, Law M, Babb JS, Rueff LE, Jacob JS, Kim S, Carson RH, Mulholland TP, Laub G, Hecht EM. 3D time-resolved MR angiography (MRA) of the carotid arteries with time-resolved imaging with stochastic trajectories: comparison 
with 3D contrast-enhanced Bolus-Chase MRA and 3D time-of-flight MRA. AJNR Am J Neuroradiol 2008;29(10):1847-1854.

8. Le Y, Nickel D, Kroeker R, Geppert C, Dale B, Kipfer H, Lin C. A Simulation Study of the Flexible TWIST View Sharing Impact on the Breast DCE MRI. ISMRM. Milan, Italy; 2014. p 6436. 


\section{Figures}

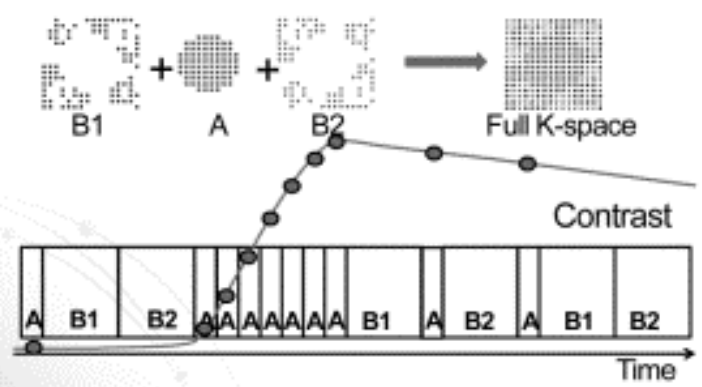

Figure 1. The timing of TWIST-Dixon-Flex acquisition in breast DCE-MRI relative to the contrast wash-in and wash-out is illustrated. The wash-out type of enhancement curve (Red) is used as an example. "Full” $k$-space acquisition is applied for the first pre-contrast measurement as well as the last measurement. Immediately after the injection of the contrast, there are six measurements with 'Center-only' $k$-space acquisition. Red dots represent the time point when the $k$-space center is acquired.

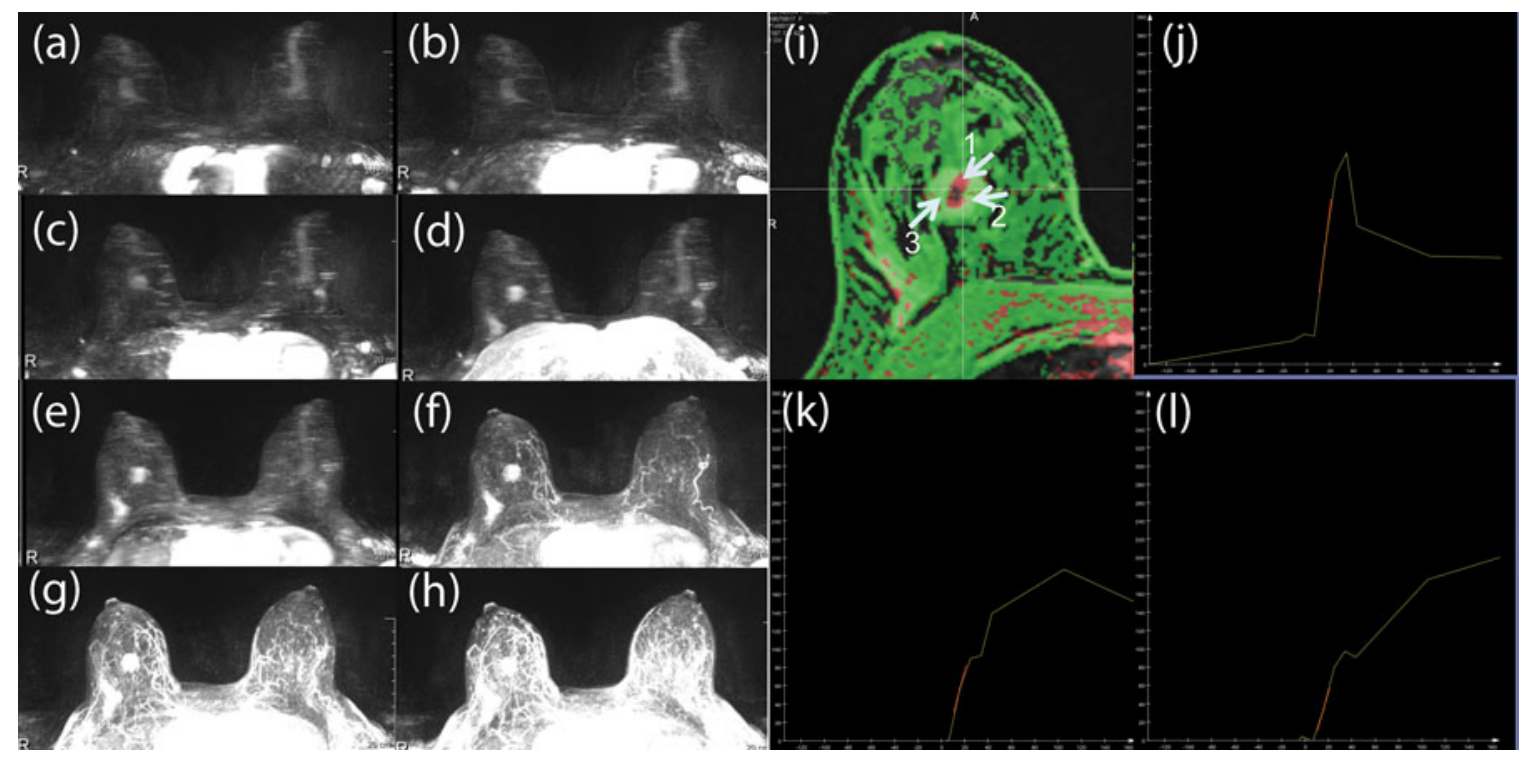

Figure 2. (a-h) Sequential Maximum Intensity Projection (MIP) images from $3^{\text {rd }}$ (a) to the $10^{\text {th }}$ (h) time points. These MIP images demonstrate the early enhancement and the change over time of a 
tumor (known invasive ductal carcinoma) with typical increasing background enhancement. In the maximum slope color map overlay (i), red color represents voxels with enhancement slope greater than $6 \%$ per second, while green color represents slope less than $6 \%$. Although 'green' voxels labeled by arrows 2 and 3 both have similar maximum slope, their overall enhancement types are different. Different voxels also reach peak enhancement at different time points: voxel 1 peaks at the $7^{\text {th }}$ time point $(\mathrm{j})$, while voxel 2 peaks at the $9^{\text {th }}$ time point $(\mathrm{k})$, and voxel 3 continues to be increasingly enhanced during the whole process (l).

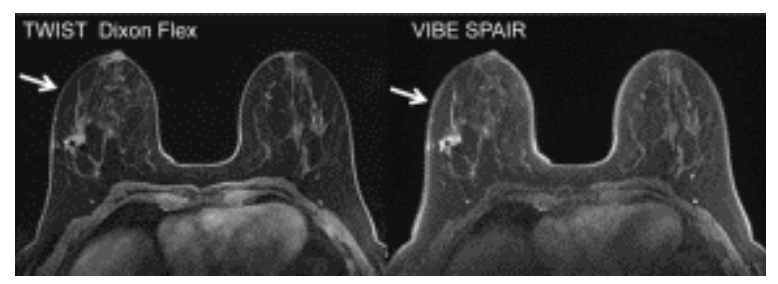

Figure 3. Comparison between TWIST-Dixon-Flex image at the peak enhancement, and VIBESPAIR image in the end of the scan, in the same patient. Note the obvious ringing artifact at the surface of the tissue in the VIBE-SPAIR image (arrow), in TWIST-Dixon-Flex image, there is much less of such artifact. 